\title{
Venous Thromboembolism in Critically Ill Cirrhotic Patients: Practices of Prophylaxis and Incidence
}

\author{
Hasan M. Al-Dorzi, ${ }^{1,2}$ Hani M. Tamim, ${ }^{3,4}$ Abdulaziz S. Aldawood, ${ }^{1,2}$ and Yaseen M. Arabi ${ }^{1,2}$ \\ ${ }^{1}$ Intensive Care Department, King Abdulaziz Medical City, Mail Code 1425, P.O. Box 22490, Riyadh 11426, Saudi Arabia \\ ${ }^{2}$ College of Medicine, King Saud Bin Abdulaziz University for Health Sciences, Riyadh 11426, Saudi Arabia \\ ${ }^{3}$ Epidemiology and Biostatistics, King Abdullah International Medical Research Center, King Saud Bin Abdulaziz University for \\ Health Sciences, P.O. Box 22490, Riyadh 11426, Saudi Arabia \\ ${ }^{4}$ Department of Internal Medicine, American University of Beirut Medical Center, P.O. Box 11-0236, Beirut 1107 2020, Lebanon
}

Correspondence should be addressed to Yaseen M. Arabi; yaseenarabi@yahoo.com

Received 12 June 2013; Revised 3 September 2013; Accepted 21 October 2013

Academic Editor: Edith Nutescu

Copyright (C) 2013 Hasan M. Al-Dorzi et al. This is an open access article distributed under the Creative Commons Attribution License, which permits unrestricted use, distribution, and reproduction in any medium, provided the original work is properly cited.

\begin{abstract}
Objectives. We compared venous thromboembolism (VTE) prophylaxis practices and incidence in critically ill cirrhotic versus noncirrhotic patients and evaluated cirrhosis as a VTE risk factor. Methods. A cohort of 798 critically ill patients followed for the development of clinically detected VTE were categorized according to the diagnosis of cirrhosis. VTE prophylaxis practices and incidence were compared. Results. Seventy-five (9.4\%) patients had cirrhosis with significantly higher INR ( $2.2 \pm 0.9$ versus $1.3 \pm 0.6$, $P<0.0001)$, lower platelet counts $(115,000 \pm 90,000$ versus $258,000 \pm 155,000 / \mu \mathrm{L}, P<0.0001)$, and higher creatinine compared to noncirrhotic patients. Among cirrhotics, 31 patients received only mechanical prophylaxis, 24 received pharmacologic prophylaxis, and $20 \mathrm{did}$ not have any prophylaxis. Cirrhotic patients were less likely to receive pharmacologic prophylaxis (odds ratio, $0.08 ; 95 \%$ confidence interval (CI), 0.04-0.14). VTE occurred in only two (2.7\%) cirrhotic patients compared to $7.6 \%$ in noncirrhotic patients $(P=0.11)$. The incidence rate was 2.2 events per 1000 patient-ICU days for cirrhotic patients and 3.6 events per 1000 patient-ICU days for noncirrhotics (incidence rate ratio, 0.61; 95\% CI, 0.15-2.52). On multivariate Cox regression analysis, cirrhosis was not associated with VTE risk (hazard ratio, 0.40; 95\% CI, 0.10-1.67). Conclusions. In critically ill cirrhotic patients, VTE incidence did not statistically differ from that in noncirrhotic patients.
\end{abstract}

\section{Background}

Chronic liver disease leads to decreased synthesis of coagulation proteins, such as factors II, VII, IX, and X, and is frequently associated with thrombocytopenia [1-3]. Whether these abnormalities make cirrhotic patients less prone to venous thromboembolism (VTE) than the general population is unclear, especially given that cirrhosis is also associated with decreased production of anticoagulation factors, such as protein $\mathrm{C}$, protein $\mathrm{S}$, and antithrombin III $[2,3]$. A population-based, case-control study found that liver disease was associated with reduced VTE risk (odds ratio (OR), 0.1; 95\% CI, 0.0-0.7) [4]. One retrospective case-control study in hospitalized cirrhotic patients found that VTE occurred in only $0.5 \%$ of patients [5], a rate that was lower than that reported in general medical patients [5]. However, more recent studies found higher VTE rates in hospitalized cirrhotic patients $(2.7-6.3 \%)$ [6, 7]. Additionally, a study of 963 cirrhotic patients and 12,405 controls admitted to a tertiary care hospital found that cirrhotics had higher (1.8\%) VTE incidence than controls in general $(0.9 \%, P=0.007)$ but lower than patients with heart failure $(7.8 \%)$, chronic kidney disease (7.1\%), and cancer (6.1\%) [8]. The study also showed that cirrhosis was not associated with VTE on multivariate analysis (OR, 0.87; 95\% CI, 0.28-2.63) [8]. None of these studies evaluated VTE exclusively in critically ill cirrhotic patients, who are likely to have higher VTE risk like other intensive care unit (ICU) patients [9]. In addition, the evidence-based VTE prophylaxis guidelines have no separate recommendation for cirrhotic patients [10-12] and caution 
against pharmacologic prophylaxis for patients with bleeding risk including those with platelet count $<50,000 / \mu \mathrm{L}$, liver failure, and international normalized ratio (INR) $>1.5$ [12]. This may make the practices of VTE prophylaxis in cirrhotic patients inconsistent and variable.

The present study described the practices of VTE prophylaxis in cirrhotic patients admitted to the ICU in relation to coagulation status and evaluated the incidence of VTE (deep venous thrombosis (DVT) or pulmonary embolism (PE)) compared to other critically ill patients and studied the outcome of VTE in this patient population.

\section{Methods}

2.1. Setting and Patients. This was a retrospective analysis of a prospective cohort of 798 consecutive patients who were admitted to the ICU of King Abdulaziz Medical City, Riyadh, from January 1, 2006, to June 30, 2008, and were followed for the occurrence of DVT or PE from ICU admission till death in the ICU or up to 5 days after ICU discharge if remained hospitalized. Patients with the following conditions were excluded from the cohort: expected ICU stay $<48$ hours, brain death on admission, Do-Not-Resuscitate order in the first 48 hours of admission, chronic systemic anticoagulation, and DVT/PE on or within 24 hours of ICU admission. The hospital was a 900-bed tertiary care center, was accredited by the Joint Commission International, and had developed its own VTE prophylaxis guidelines, which mostly followed the American College of Chest Physicians evidence-based clinical practice guidelines $[10,13]$. The ICU was a 21-bed closed medical surgical unit staffed with board certified intensivists 24 hours a day, 7 days per week [14]. In this ICU, VTE prophylaxis was ordered by the treating intensivist as pharmacologic prophylaxis (unfractionated heparin or lowmolecular-weight heparin) and/or mechanical prophylaxis in the form of graduated compression stocking (GCS) and/or intermittent pneumatic compression (IPC) devices as part of admission order set. Investigation for VTE was performed when clinically suspected by the treating intensivist. Venous duplex ultrasound of the extremities was performed to diagnose DVT and helical chest computed tomography to evaluate PE.

2.2. Data Collection. In the present study, a trained research physician evaluated and followed the patients in the cohort almost on a daily basis for up to 30 days in the ICU and five days after ICU discharge. The required data, such as laboratory results, VTE prophylaxis practices, and outcomes, were recorded in case report forms. Charts were also reviewed to obtain additional clinical information, such as past medical history and VTE risk factors. Patients known to have liver cirrhosis, based on their documented past medical history, were identified and compared to all other patients for the incidence of VTE (DVT or PE). In addition, the following information was noted: age, gender, body mass index, reason of ICU admission (postoperative or medical), Acute Physiology and Chronic Health Evaluation (APACHE) II score [15], requirement for mechanical ventilation and duration of mechanical ventilation, admission creatinine, bilirubin, ammonia, albumin, platelet count, INR of the prothrombin time and partial thromboplastin time (PTT), use of mechanical prophylaxis in the form of graduated GCS or IPC, use of pharmacologic prophylaxis in the form of unfractionated and low-molecular-weight heparin, and transfusion of blood products. Other studied outcomes included the duration of mechanical ventilation, length of stay in the ICU and the hospital, and ICU and hospital mortality. Patients were also categorized according to clinically relevant cutoffs of platelet count (cutoff of 50,000/ $\mu \mathrm{L}$ ), INR (cutoff of 2.0), and PTT (cutoff of 45 seconds).

2.3. Statistical Analysis. Data were analyzed using SAS software (version 9.0; SAS Institute, Cary, NC, USA). Continuous data were presented as means and standard deviation (SD), whereas categorical variables were summarized as numbers and percentages (\%). Chi-square or Fisher's exact test was used to evaluate differences in categorical variables between cirrhotic and noncirrhotic patients. Similarly, Student's $t$ test was used to assess differences in continuous variables. We calculated the incidence rates for VTE in cirrhotic and noncirrhotics and reported the incidence rate ratio with 95\% confidence interval (CI). Cox proportional regression analysis was performed to determine if cirrhosis was an independent risk factor for VTE in critically ill patients adjusting for clinically significant factors and imbalances in baseline characteristics, which were age, gender, creatinine, use of low-molecular-weight heparin, platelet count, INR, admission diagnosis, trauma, femur fracture, presence of central line, sepsis, spinal cord injury status, malignancy, surgery, previous PE or DVT, and stroke. Its result was presented as hazard ratio with $95 \% \mathrm{CI}$.

\section{Results}

3.1. General Characteristics and VTE Risk Factors. Out of the 798 patients included in the cohort, 75 (9.4\%) had liver cirrhosis. Table 1 describes their characteristics and VTE risk factors compared to noncirrhotic patients. Patients with cirrhosis were older, had higher APACHE II score $(30 \pm 8$ versus $23 \pm 9, P<0.0001$ ), and were less likely to be admitted postoperatively or after polytrauma. In addition, they were less likely to have a diagnosis of stroke and to be functionally independent and mobile at home before admission. However, they had similar prevalence of malignancy and requirement for mechanical ventilation. On admission to the ICU, they also had significantly higher INR $(2.2 \pm 0.9$ versus $1.3 \pm$ $0.6, P<0.0001)$, lower platelet count $(115,000 \pm 90,000$ versus $258,000 \pm 155,000 / \mu \mathrm{L}, P<0.0001$ ), and higher serum bilirubin and creatinine.

3.2. Practices of VTE Prophylaxis. Table 2 summarizes the practices of VTE prophylaxis according to cirrhosis status. Forty-three (57\%) cirrhotic patients received mechanical prophylaxis in the form of IPC and/or GCS, with 31 (41\%) cirrhotic patients receiving only mechanical prophylaxis. Use of mechanical prophylaxis was similar in cirrhotic 
TABLE 1: Characteristics of patients with and without liver cirrhosis.

\begin{tabular}{|c|c|c|c|}
\hline & $\begin{array}{l}\text { Cirrhotic patients } \\
\quad N=75\end{array}$ & $\begin{array}{l}\text { Noncirrhotic patients } \\
\qquad N=723\end{array}$ & $P$ value \\
\hline Age (years), mean $\pm S D$ & $58.8 \pm 14.3$ & $49.3 \pm 21.6$ & $<0.0001$ \\
\hline Male gender, $N(\%)$ & $37(49.3)$ & $498(68.9)$ & 0.0006 \\
\hline Body mass index $\left(\mathrm{Kg} / \mathrm{m}^{2}\right)$, mean $\pm \mathrm{SD}$ & $29.4 \pm 7.8$ & $28.4 \pm 10.4$ & 0.31 \\
\hline APACHE II score, mean \pm SD & $29.6 \pm 8.2$ & $23.4 \pm 8.9$ & $<0.0001$ \\
\hline Admission GCS score, mean \pm SD & $9.2 \pm 4.1$ & $8.5 \pm 4.1$ & 0.19 \\
\hline \multicolumn{4}{|l|}{ Reason for ICU admission: } \\
\hline Respiratory, $N(\%)$ & $10(13.3)$ & $159(22.0)$ & \multirow{6}{*}{$<0.0001$} \\
\hline Cardiovascular, $N(\%)$ & $45(60.0)$ & $201(27.8)$ & \\
\hline Neurological, $N(\%)$ & $1(1.3)$ & $56(7.8)$ & \\
\hline Other medical, $N(\%)$ & $17(22.7)$ & $19(2.6)$ & \\
\hline Nonoperative trauma, $N(\%)$ & $1(1.3)$ & $122(16.9)$ & \\
\hline Postoperative, $N(\%)$ & $1(1.3)$ & $166(23.0)$ & \\
\hline Bedridden before admission, $N(\%)$ & $54(72.0)$ & $340(47.0)$ & $<0.0001$ \\
\hline Congestive heart failure, $N(\%)$ & $2(2.6)$ & $36(5.0)$ & 0.57 \\
\hline Previous stroke, $N(\%)$ & $4(5.3)$ & $102(14.1)$ & 0.03 \\
\hline Active malignancy, $N(\%)$ & $7(9.3)$ & $87(12.0)$ & 0.49 \\
\hline Femur fracture, $N(\%)$ & $1(1.3)$ & $51(7.0)$ & 0.08 \\
\hline Spinal cord injury, $N(\%)$ & $0(0)$ & $20(2.8)$ & 0.24 \\
\hline Previous history of VTE, $N(\%)$ & $0(0)$ & $12(1.7)$ & 0.62 \\
\hline Sepsis on admission, $N(\%)$ & $55(73.3)$ & $266(36.8)$ & $<0.0001$ \\
\hline Mechanical ventilation on admission, $N(\%)$ & $62(82.7)$ & $625(86.4)$ & 0.37 \\
\hline Femoral central venous catheter, $N(\%)$ & $47(62.7)$ & $295(40.8)$ & 0.0003 \\
\hline $\begin{array}{l}\text { Internal jugular or subclavian central venous } \\
\text { catheter, } N(\%)\end{array}$ & $50(66.7)$ & $475(65.7)$ & 0.87 \\
\hline Admission creatinine $(\mu \mathrm{mol} / \mathrm{L})$, mean $\pm \mathrm{SD}$ & $208 \pm 149$ & $154 \pm 143$ & 0.002 \\
\hline Admission lactate $(\mathrm{mmol} / \mathrm{L})$, mean $\pm \mathrm{SD}$ & $4.5 \pm 3.8$ & $3.1 \pm 3.1$ & 0.004 \\
\hline Admission bilirubin $(\mu \mathrm{mol} / \mathrm{L})$, mean $\pm \mathrm{SD}$ & $261 \pm 272$ & $34 \pm 62$ & $<0.0001$ \\
\hline Admission platelet count $/ \mu \mathrm{L}$, mean \pm SD & $115,000 \pm 90,000$ & $258,000 \pm 155,000$ & $<0.0001$ \\
\hline Admission INR, mean \pm SD & $2.2 \pm 0.9$ & $1.3 \pm 0.6$ & $<0.0001$ \\
\hline Admission PTT (seconds), mean \pm SD & $44.1 \pm 62.0$ & $54.3 \pm 35.9$ & 0.07 \\
\hline
\end{tabular}

APACHE: Acute Physiology and Chronic Health Evaluation; GCS: Glasgow Coma Scale; ICU: intensive care unit; INR: International Normalized Ratio; PTT: partial thromboplastin time; SD: standard deviation.

and noncirrhotic patients $(57.3 \%$ and $50.6 \%$, resp., $P=$ 0.27 ) (OR, 1.31; 95\% CI, 0.81-2.12). However, more (41.3\%) cirrhotics received only mechanical prophylaxis compared to noncirrhotics $(7.7 \%, P<0.0001)$. For pharmacologic prophylaxis, cirrhotic patients were less likely to receive pharmacologic prophylaxis for DVT prophylaxis (odds ratio, 0.08; 95\% CI, 0.04-0.14) such that only $22(29.3 \%)$ patients received unfractionated heparin compared to $66.3 \%$ in noncirrhotic patients $(P<0.0001)$ and two $(2.7 \%)$ patients received lowmolecular-weight heparin compared to $31.1 \%$ of noncirrhotic patients $(P<0.0001)$. Of note, more $(26.7 \%)$ cirrhotic patients did not receive any form of prophylaxis compared to noncirrhotics (7.2\%, $P<0.0001)$. Moreover, cirrhotic patients had shorter duration of pharmacologic prophylaxis $(5.1 \pm 4.2$ versus $10.4 \pm 7.6$ days for noncirrhotics, $P<$ 0.0001 ) and more days in the ICU without pharmacologic prophylaxis $(8.4 \pm 7.0$ versus $4.1 \pm 6.1$ days for noncirrhotics, $P<0.0001)$.

Figure 1 describes the use of pharmacologic prophylaxis in cirrhotic patients according to platelet count $(<$ versus $\geq 50,000 / \mu \mathrm{L})$, INR ( $<$ versus $\geq 2.0$ ), and PTT ( $<$ versus $\geq 45$ seconds) on ICU admission. Pharmacologic prophylaxis was used less often in patients with INR $\geq 2.0(P=$ $0.003)$ and PTT $\geq 45$ seconds $(P<0.001)$. In cirrhotic patients, those who were given pharmacologic prophylaxis had higher platelet counts $(146,000 \pm 100,000 / \mu \mathrm{L}$ versus $99,000 \pm 82,000 / \mu \mathrm{L}, P=0.04)$ and lower PTT $(39 \pm 11$ seconds versus $61 \pm 41$ seconds, $P=0.0008)$ and INR $(1.7 \pm 0.5$ versus $2.4 \pm 1.1, P=0.003)$ and received less transfusion of blood products (RBC transfusion: $2.2 \pm 2.8$ units versus $5.3 \pm 5.7$ units, $P=0.002$; platelet transfusion: $5.0 \pm 9.0$ units versus $12.9 \pm 20.9$ units, $P=0.02$; fresh frozen plasma: $7.1 \pm 9.2$ units 
TABLE 2: Practices of VTE prophylaxis in cirrhotics and noncirrhotic critically ill patients.

\begin{tabular}{|c|c|c|c|}
\hline & $\begin{array}{l}\text { Cirrhotic patients } \\
\qquad N=75\end{array}$ & $\begin{array}{l}\text { Noncirrhotic patients } \\
\qquad N=723\end{array}$ & $P$ value \\
\hline Use of mechanical prophylaxis, $N(\%)$ & $43(57.3)$ & $366(50.6)$ & 0.27 \\
\hline Intermittent pneumatic compression & $23(30.6)$ & $232(32.1)$ & 0.86 \\
\hline Graduated compression stockings & $22(29.3)$ & $175(24.2)$ & 0.29 \\
\hline Mechanical prophylaxis only & $31(41.3)$ & $56(7.7)$ & $<0.0001$ \\
\hline Use of pharmacologic prophylaxis, $N(\%)$ & $24(32.0)$ & $615(85.1)$ & $<0.0001$ \\
\hline Unfractionated heparin & $22(29.3)$ & $479(66.3)$ & $<0.0001$ \\
\hline Low-molecular-weight heparin & $2(2.7)$ & $225(31.1)$ & $<0.0001$ \\
\hline Pharmacologic prophylaxis only & $12(16.0)$ & $305(42.2)$ & $<0.0001$ \\
\hline No VTE prophylaxis, $N$ (\%) & $20(26.7)$ & $52(7.2)$ & $<0.0001$ \\
\hline $\begin{array}{l}\text { Use of both mechanical and pharmacologic } \\
\text { prophylaxis simultaneously, } N(\%)\end{array}$ & $12(16.0)$ & $310(42.9)$ & $<0.0001$ \\
\hline 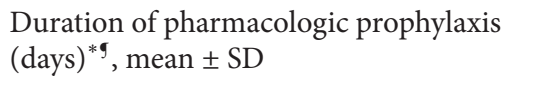 & $5.1 \pm 4.2$ & $10.4 \pm 7.6$ & $<0.0001$ \\
\hline \multicolumn{4}{|l|}{$\begin{array}{l}\text { Duration of mechanical prophylaxis (days), } \\
\text { mean } \pm \text { SD }\end{array}$} \\
\hline Intermittent pneumatic compression & $2.4 \pm 4.7$ & $3.3 \pm 6.3$ & 0.13 \\
\hline Graduated compression stockings & $2.9 \pm 5.4$ & $2.3 \pm 5.2$ & 0.41 \\
\hline $\begin{array}{l}\text { Stay in the ICU without pharmacologic } \\
\text { prophylaxis }\end{array}$ & $8.4 \pm 7.0$ & $4.1 \pm 6.1$ & $<0.0001$ \\
\hline
\end{tabular}

ICU: intensive care unit; SD: standard deviation.

${ }^{*}$ For patients who received pharmacologic prophylaxis.

'Data on thromboprophylaxis were obtained for a maximum of 30 days of ICU stay.

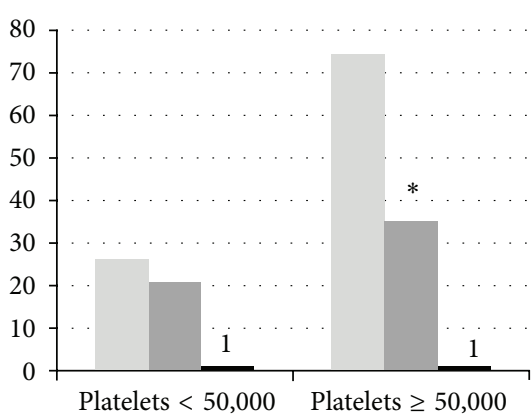

Percentage of cirrhotic patients

- Percentage on anticoagulant prophylaxis

- Number of patients with VTE

$$
\begin{aligned}
& { }^{*} P=0.25 \\
& { }^{* *} P=0.003 \\
& { }^{* * *} P<0.001
\end{aligned}
$$

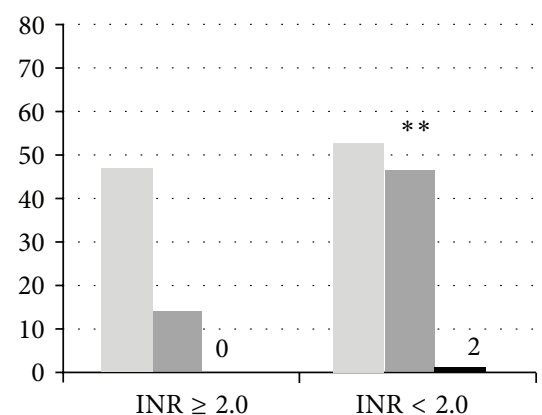

- Percentage of cirrhotic patients

- Percentage on anticoagulant prophylaxis

- Number of patients with VTE

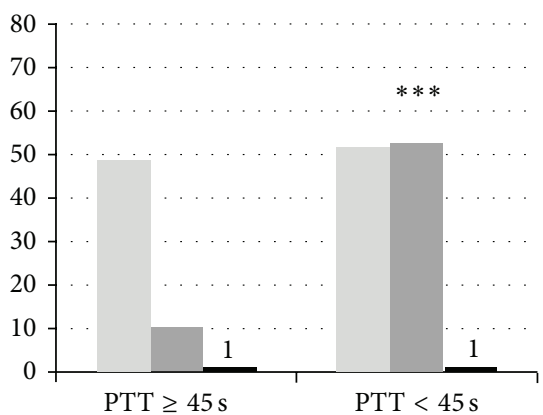

Percentage of cirrhotic patients

Percentage on anticoagulant prophylaxis

- Number of patients with VTE

FIGURE 1: The relationship between the use of pharmacologic prophylaxis and the admission values of platelet count (per $\mu \mathrm{L})$, INR, and PTT in cirrhotic patients.

versus $15.2 \pm 12.3$ units, $P=0.006$; and cryoprecipitates: 1.1 \pm 3.0 units versus $4.7 \pm 8.2$ units, $P=0.007$ ) compared to patients not given pharmacologic prophylaxis.

The most common reason for not using pharmacologic prophylaxis in cirrhotic patients was bleeding risk in 55 (73.3\%) patients. In noncirrhotic patients, the reasons were more variable and included recent surgery in 137 (18.9\%) patients, intracranial hemorrhage in 123 (17.0\%) patients, and other bleeding risks in $142(19.6 \%)$ patients. The most common reasons for not using mechanical prophylaxis were the use of pharmacologic prophylaxis in 12 cirrhotic and 327 noncirrhotic patients and lower extremity fracture in 21 noncirrhotic patients.

3.3. Incidence of VTE during ICU Stay. Table 3 describes the outcomes of patients according to liver cirrhosis status. 
TABLE 3: Outcomes of patients in the cohort according to cirrhosis status.

\begin{tabular}{lccc}
\hline & Cirrhotic patients & Noncirrhotic patients & N $=723$ \\
& $N=75$ & $55(7.6)$ & \\
Venous thromboembolism, $N(\%)$ & $2(2.7)$ & $27(3.7)$ & 0.11 \\
$\quad$ Deep venous thrombosis alone & $2(2.7)$ & $24(3.3)$ & 0.64 \\
$\quad$ Pulmonary embolism alone & $0(0)$ & $4(0.6)$ & 0.11 \\
$\quad$ Deep venous thrombosis and pulmonary embolism & $0(0)$ & $9.9 \pm 12.7$ & 0.52 \\
Duration of MV (days) & $8.3 \pm 6.9$ & $16.9 \pm 31.3$ & 0.09 \\
ICU LOS (days) & $10.2 \pm 7.7$ & $75.9 \pm 125.1$ & $<0.0001$ \\
Hospital LOS (days) & $27.7 \pm 21.2$ & $123(17.0)$ & $<0.0001$ \\
ICU mortality, $N$ (\%) & $48(64.0)$ & $226(31.3)$ & $<0.0001$ \\
Hospital mortality, $N(\%)$ & $60(80.0)$ & & $<0.0001$ \\
\hline
\end{tabular}

ICU: intensive care unit; LOS: length of stay; MV: mechanical ventilation.

VTE was diagnosed in only two $(2.7 \%)$ cirrhotic patients compared to $55(7.6 \%)$ noncirrhotic patients $(P=0.11)$. On multivariate Cox regression analysis, cirrhosis was not an independent risk factor for VTE (hazard ratio, 0.40 ; $95 \%$ CI, $0.10-1.67)$. The incidence rate ratio was 2.2 events per 1000 patient-days for cirrhotic patients and 3.6 events per 1000 patient-days for noncirrhotics yielding an incidence rate ratio of 0.61 (95\% CI, 0.15-2.52). The two cirrhotic patients who had VTE were females with mean INR $=1.25$ (1.1 and 1.4) and both had femoral central lines. One had DVT on the fifth ICU day and the other on the 13th day. For VTE prophylaxis, one was on unfractionated heparin with normal platelet count and the other was only on mechanical prophylaxis because of platelet count $<50,000 / \mu \mathrm{L}$. None of the cirrhotic patients was diagnosed to have PE compared to 28 (3.9\%) patients without cirrhosis $(P=0.08)$.

Of note, patients with liver cirrhosis had significantly shorter duration of stay in the ICU (10 \pm 8 versus $17 \pm 31$ days) and higher mortality in the ICU ( $64 \%$ versus $17 \%, P<0.0001$ and hospital ( $80 \%$ versus $31 \%, P<0.0001)$ ). The two cirrhotic patients who had DVT died in the ICU.

\section{Discussion}

The main findings of the present study were the following: the majority $(73 \%)$ of critically ill cirrhotic patients received at least one form of VTE prophylaxis, but they were less likely to receive pharmacologic prophylaxis than noncirrhotic patients; VTE incidence did not differ between cirrhotic and noncirrhotic critically ill patients when VTE was investigated upon clinical suspicion and cirrhosis was not an independent predictor of the occurrence of VTE in the ICU.

Cirrhotic patients have decreased synthesis of procoagulant proteins, which results in the prolongation of the prothrombin time, and frequently have thrombocytopenia [1-3]. Hence, they are frequently considered to be autoanticoagulated. However, these patients have a concomitant reduced synthesis of anticoagulant factors [2,3], may experience poor blood flow and vasculopathy [2,3], and have high incidence of antiphospholipid antibodies [16], all of which increase thrombosis risk. Studies on thromboprophylaxis in cirrhotic patients are lacking. In fact, severe liver disease was an exclusion criterion for many thromboprophylaxis trials in medical patients [17-19]. This may have resulted in evidence-based VTE prophylaxis guidelines having no separate recommendations for patients with liver cirrhosis [10-12, 20], although they are frequently considered as patients with increased bleeding risk $[11,12]$. This may make clinicians reluctant to use pharmacologic VTE prophylaxis. This was observed in multiple studies. Northup and colleagues found that only $21 \%$ of 113 hospitalized cirrhotic patients received DVT prophylaxis [5]. Dabbagh and colleagues similarly found that only $25.3 \%$ of 190 hospitalized cirrhotic patients received DVT prophylaxis (9\% pharmacologic and $16.3 \%$ mechanical) [7]. In a previous study of 226 cirrhotic patients admitted to our hospital, approximately $76 \%$ of the cirrhotic patients received neither pharmacologic nor mechanical DVT prophylaxis [6]. The present study evaluated cirrhotic patients who developed critical illness and were managed with interventions that further increase VTE risk. We have found that the practices of VTE prophylaxis in cirrhotic ICU patients were significantly different from those in noncirrhotics. In contrast to other studies, $73 \%$ of cirrhotic patients received at least one form of prophylaxis in our study. Pharmacologic prophylaxis was used less often especially in patients with higher INR and PTT. This was also observed in other studies.

Studies that evaluated VTE incidence in cirrhotic patients showed variable findings. A population-based case-control study over 15 years between 1976 and 1990 found that serious liver disease was associated with a $90 \%$ decrease in the risk for VTE [4]. Northup et al. conducted a retrospective cohort study over an eight-year period, identified 113 hospitalized patients with cirrhosis, and found a VTE incidence of $0.5 \%$ [5]. Gulley et al. conducted a case-control study of medical patients at a tertiary care hospital, identified patients with newly diagnosed VTE based on the related ICD-9 codes, and confirmed the diagnosis by positive computerized tomography, positive Doppler ultrasound of the extremities, and/or high probability V/Q scan [8]. They found a $1.8 \%$ incidence of VTE in cirrhotic patients compared to $0.9 \%$ in the controls [8]. However, other studies have found higher VTE incidence in hospitalized cirrhotic patients $[7,21]$. In 
a recently published observational study of 190 hospitalized cirrhotic patients admitted to a tertiary university hospital over a seven-year period, the incidence of new VTE was $6.3 \%$ [7]. In a study from Asia where viral hepatitis is the predominant cause of cirrhosis, the incidence of VTE in hospitalized cirrhotic patients was $4.7 \%$ [21]. In a study from our hospital, $2.7 \%$ of 226 cirrhotic patients admitted over one-year period developed VTE, all of which were DVT [6]. The present study was on a different population and for a different follow-up period and found an incidence rate of 2.2 VTE events per 1000 patient-days for cirrhotic patients. This incidence rate was not significantly different than that of noncirrhotic patients. We found that cirrhotic patients who got pharmacologic prophylaxis had higher platelet count and more favorable coagulation profile than cirrhotic patients without pharmacologic prophylaxis suggesting that these factors are important when clinicians decide on the VTE prophylaxis method. Additionally, the same patients had lower needs for blood transfusion during ICU stay, possibly for the same reasons.

The majority of critically ill patients have multiple VTE risk factors [22]. Most of these risk factors apply to cirrhotic patients during critical illness. PTT and serum albumin have been found to independently predict VTE in cirrhotic patients [8]. However, Dabbagh and colleagues showed that the level of INR did not affect VTE incidence [7]. In the present study, the only two cirrhotic patients who developed VTE had their INR within the normal range $(<1.5)$. They also had femoral central venous catheters, which increases thrombosis risk through multiple mechanisms [23]. It is not surprising that the femoral vein was frequently used for central venous access in cirrhotic patients as these patients were thought to be at high risk of bleeding. The femoral route has several potential advantages. The site is compressible if bleeding occurs and it can facilitate successful resuscitation in acute decompensation [24].

The present study has strengths and limitations. Among the strengths are the prospective data collection by a trained research physician and the presence of evidence-based hospital VTE prophylaxis guidelines and order set. Limitations include the post hoc analysis, the small number of patients with cirrhosis, the low number of VTE events in this group, conducting the study at a single center and investigating VTE only when it was clinically suspected, which may have led to diagnosis bias. It is possible that physicians were less likely to suspect VTE or perform workup in cirrhotic patients because of the perception that these patients have low likelihood of having VTE due to coagulopathy and also due to poor prognosis. Such bias leads to underestimation of the true VTE risk in cirrhotic patients. So subclinical VTE might have been missed. The incidence of VTE in our cohort of 798 consecutive critically ill patients was $7.1 \%$. Other prospective ICU cohorts have shown different VTE incidence rates [25-27]. Hirsch et al. performed Doppler ultrasound imaging twice weekly in the ICU and once within one week of ICU discharge, had only $61 \%$ of patients given prophylaxis, and found a DVT incidence of 33\% [26]. In Ibrahim et al's study, Doppler ultrasound of the lower and upper extremities was performed every seven days, all patients received VTE prophylaxis, and the DVT incidence was $23.6 \%$ [27]. Cook et al. performed Doppler ultrasound of lower extremities within 48 hours of ICU admission and then twice weekly thereafter, used VTE prophylaxis for all patients (pharmacologic prophylaxis for $92.8 \%$ and mechanical for the other $7.2 \%$ ), and found a DVT incidence of 9.6\% [25]. The difference in DVT incidence between the present study and the other cohort studies is mainly related to the fact that VTE evaluation was performed when clinically suspected in the present study compared to scheduled DVT surveillance in the other studies.

In conclusion, we have found that pharmacologic prophylaxis was administered less often in critically ill cirrhotic patients compared to noncirrhotic patients, but $73 \%$ of cirrhotic patients received at least one form of VTE prophylaxis. We also found that VTE incidence in cirrhotic patients was not statistically different from that of other critically ill patients. We think that all critically ill cirrhotic patients should receive VTE prophylaxis. Those with important risk factors for bleeding should receive mechanical prophylaxis, while others can be considered for pharmacologic prophylaxis after careful consideration of bleeding risk.

\section{Conflict of Interests}

The authors declare that they have no conflict of interests.

\section{Acknowledgment}

The study was sponsored in part by an unrestricted grant from Sanofi-Aventis.

\section{References}

[1] F. N. Bashour, J. C. Teran, and K. D. Mullen, "Prevalence of peripheral blood cytopenias (hypersplenism) in patients with nonalcoholic chronic liver disease," The American Journal of Gastroenterology, vol. 95, no. 10, pp. 2936-2939, 2000.

[2] M. Senzolo, P. Burra, E. Cholongitas, and A. K. Burroughs, "New insights into the coagulopathy of liver disease and liver transplantation," World Journal of Gastroenterology, vol. 12, no. 48, pp. 7725-7736, 2006.

[3] A. Tripodi and P. M. Mannucci, "The coagulopathy of chronic liver disease," The New England Journal of Medicine, vol. 365, no. 2, pp. 147-156, 2011.

[4] J. A. Heit, M. D. Silverstein, D. N. Mohr, T. M. Petterson, W. M. O'Fallon, and L. J. Melton III, "Risk factors for deep vein thrombosis and pulmonary embolism: a population-based casecontrol study," Archives of Internal Medicine, vol. 160, no. 6, pp. 809-815, 2000.

[5] P. G. Northup, M. M. McMahon, A. P. Ruhl et al., "Coagulopathy does not fully protect hospitalized cirrhosis patients from peripheral venous thromboembolism," The American Journal of Gastroenterology, vol. 101, no. 7, pp. 1524-1528, 2006.

[6] A. Aldawood, Y. Arabi, A. Aljumah et al., "The incidence of venous thromboembolism and practice of deep venous thrombosis prophylaxis in hospitalized cirrhotic patients," Thrombosis Journal, vol. 9, article 1, 2011. 
[7] O. Dabbagh, A. Oza, S. Prakash, R. Sunna, and T. M. Saettele, "Coagulopathy does not protect against venous thromboembolism in hospitalized patients with chronic liver disease," Chest, vol. 137, no. 5, pp. 1145-1149, 2010.

[8] D. Gulley, E. Teal, A. Suvannasankha, N. Chalasani, and S. Liangpunsakul, "Deep vein thrombosis and pulmonary embolism in cirrhosis patients," Digestive Diseases and Sciences, vol. 53, no. 11, pp. 3012-3017, 2008.

[9] T. L. Ortel, "Acquired thrombotic risk factors in the critical care setting," Critical Care Medicine, vol. 38, no. 2, pp. S43-S50, 2010.

[10] W. H. Geerts, D. Bergqvist, G. F. Pineo et al., "Prevention of venous thromboembolism: American College of Chest Physicians evidence-based clinical practice guidelines (8th edition)," Chest, vol. 133, no. 6, pp. 381S-453S, 2008.

[11] A. Qaseem, R. Chou, L. L. Humphrey, M. Starkey, and P. Shekelle, "Venous thromboembolism prophylaxis in hospitalized patients: a clinical practice guideline from the American College of Physicians," Annals of Internal Medicine, vol. 155, no. 9, pp. 625-632, 2011.

[12] S. R. Kahn, W. Lim, A. S. Dunn et al., "Prevention of VTE in nonsurgical patients. Antithrombotic therapy and prevention of thrombosis, 9th ed: American College of Chest Physicians evidence-based clinical practice guidelines," Chest, vol. 141, no. 2, pp. e195S-e226S, 2012.

[13] W. H. Geerts, G. F. Pineo, J. A. Heit et al., "Prevention of venous thromboembolism: The 17th ACCP Conference on Antithrombotic and Thrombolytic Therapy," Chest, vol. 126, no. 3, pp. 338S-400S, 2004.

[14] Y. Arabi, A. Alshimemeri, and S. Taher, "Weekend and weeknight admissions have the same outcome of weekday admissions to an intensive care unit with onsite intensivist coverage," Critical Care Medicine, vol. 34, no. 3, pp. 605-611, 2006.

[15] W. A. Knaus, E. A. Draper, D. P. Wagner, and J. E. Zimmerman, "APACHE II: a severity of disease classification system," Critical Care Medicine, vol. 13, no. 10, pp. 818-829, 1985.

[16] R. Rolla, D. Vay, E. Mottaran et al., "Antiphospholipid antibodies associated with alcoholic liver disease specifically recognise oxidised phospholipids," Gut, vol. 49, no. 6, pp. 852-859, 2001.

[17] B. Gårdlund, "Randomised, controlled trial of low-dose heparin for prevention of fatal pulmonary embolism in patients with infectious diseases," The Lancet, vol. 347, no. 9012, pp. 1357-1361, 1996.

[18] H. Riess, S. Haas, U. Tebbe et al., "A randomized, doubleblind study of certoparin vs. unfractionated heparin to prevent venous thromboembolic events in acutely ill, non-surgical patients: CERTIFY Study," Journal of Thrombosis and Haemostasis, vol. 8, no. 6, pp. 1209-1215, 2010.

[19] F. Fraisse, L. Holzapfel, J.-M. Coulaud et al., "Nadroparin in the prevention of deep vein thrombosis in acute decompensated COPD. The Association of Non-University Affiliated Intensive Care Specialist Physicians of France," American Journal of Respiratory and Critical Care Medicine, vol. 161, no. 4, part 1, pp. 1109-1114, 2000.

[20] G. P. Clagett, F. A. Anderson Jr., W. Geerts et al., "Prevention of venous thromboembolism," Chest, vol. 114, no. 5, pp. 531S-560S, 1998.

[21] C. R. A. Lesmana, S. Inggriani, L. Cahyadinata, and L. A. Lesmana, "Deep vein thrombosis in patients with advanced liver cirrhosis: a rare condition?" Hepatology International, vol. 4, no. 1, pp. 433-438, 2010.
[22] W. Geerts, D. Cook, R. Selby, and E. Etchells, "Venous thromboembolism and its prevention in critical care," Journal of Critical Care, vol. 17, no. 2, pp. 95-104, 2002.

[23] R. S. Boersma, K.-S. G. Jie, A. Verbon, E. C. M. van Pampus, and H. C. Schouten, "Thrombotic and infectious complications of central venous catheters in patients with hematological malignancies," Annals of Oncology, vol. 19, no. 3, pp. 433-442, 2008.

[24] G. M. Joynt, J. Kew, C. D. Gomersall, V. Y. F. Leung, and E. K. H. Liu, "Deep venous thrombosis caused by femoral venous catheters in critically III adult patients," Chest, vol. 117, no. 1, pp. 178-183, 2000.

[25] D. Cook, M. Crowther, M. Meade et al., "Deep venous thrombosis in medical-surgical critically ill patients: prevalence, incidence, and risk factors," Critical Care Medicine, vol. 33, no. 7, pp. 1565-1571, 2005.

[26] D. R. Hirsch, E. P. Ingenito, and S. Z. Goldhaber, "Prevalence of deep venous thrombosis among patients in medical intensive care," Journal of the American Medical Association, vol. 274, no. 4, pp. 335-337, 1995.

[27] E. H. Ibrahim, M. Iregui, D. Prentice, G. Sherman, M. H. Kollef, and W. Shannon, "Deep vein thrombosis during prolonged mechanical ventilation despite prophylaxis," Critical Care Medicine, vol. 30, no. 4, pp. 771-774, 2002. 


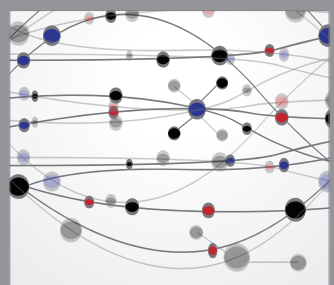

The Scientific World Journal
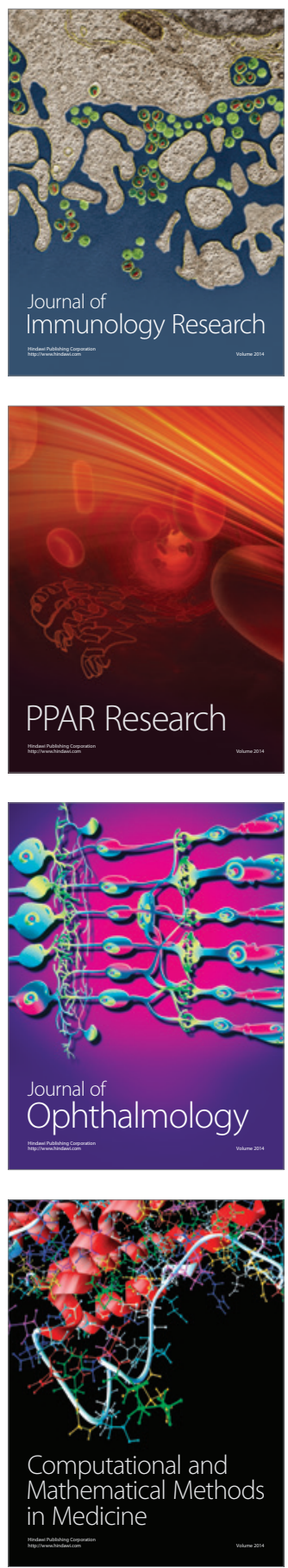

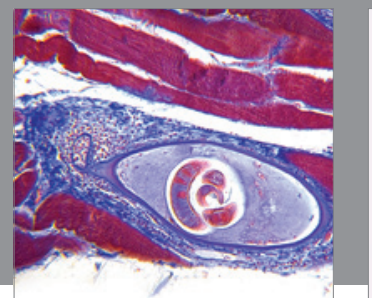

Gastroenterology

Research and Practice
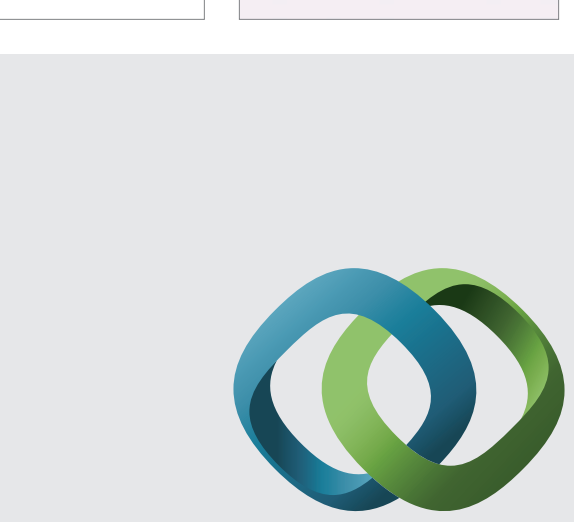

\section{Hindawi}

Submit your manuscripts at

http://www.hindawi.com
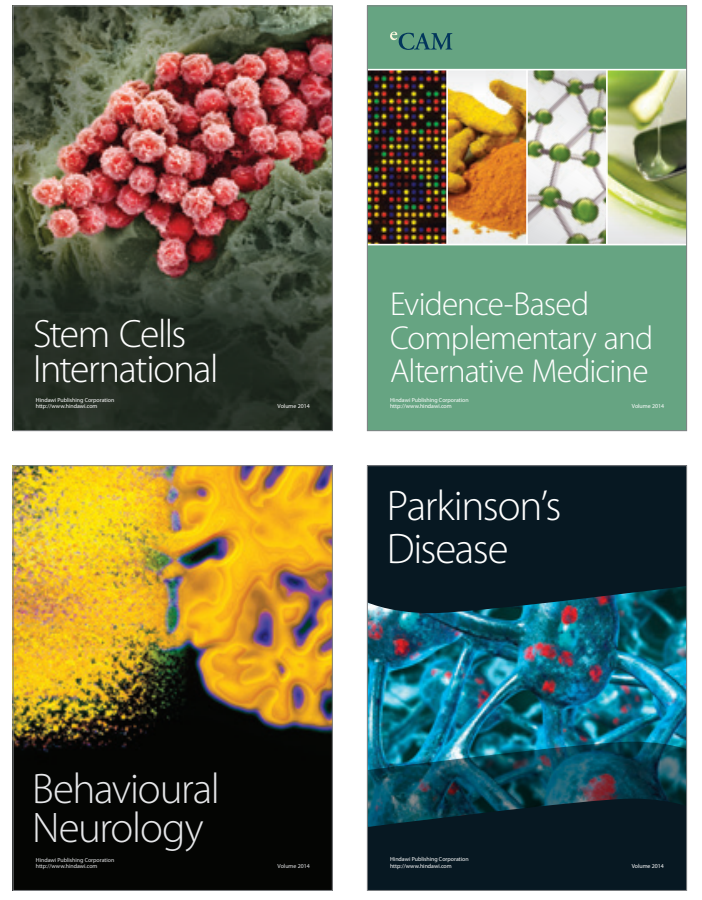
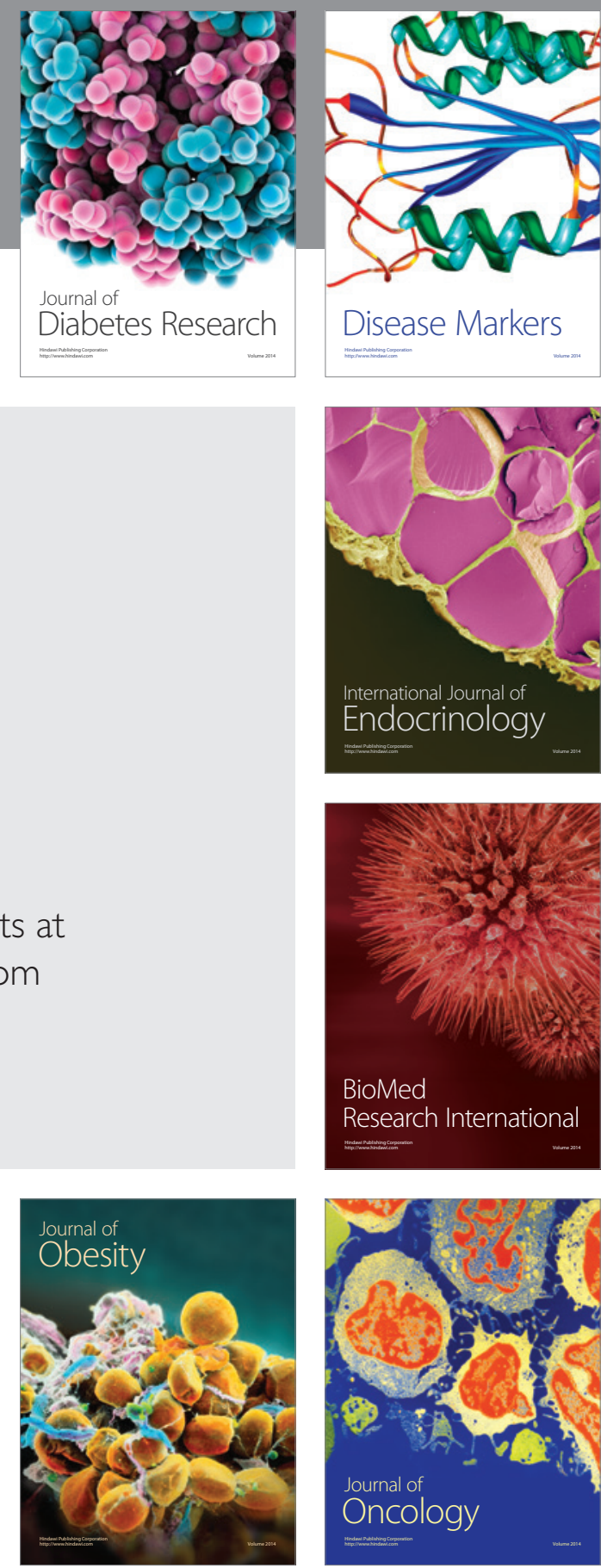

Disease Markers
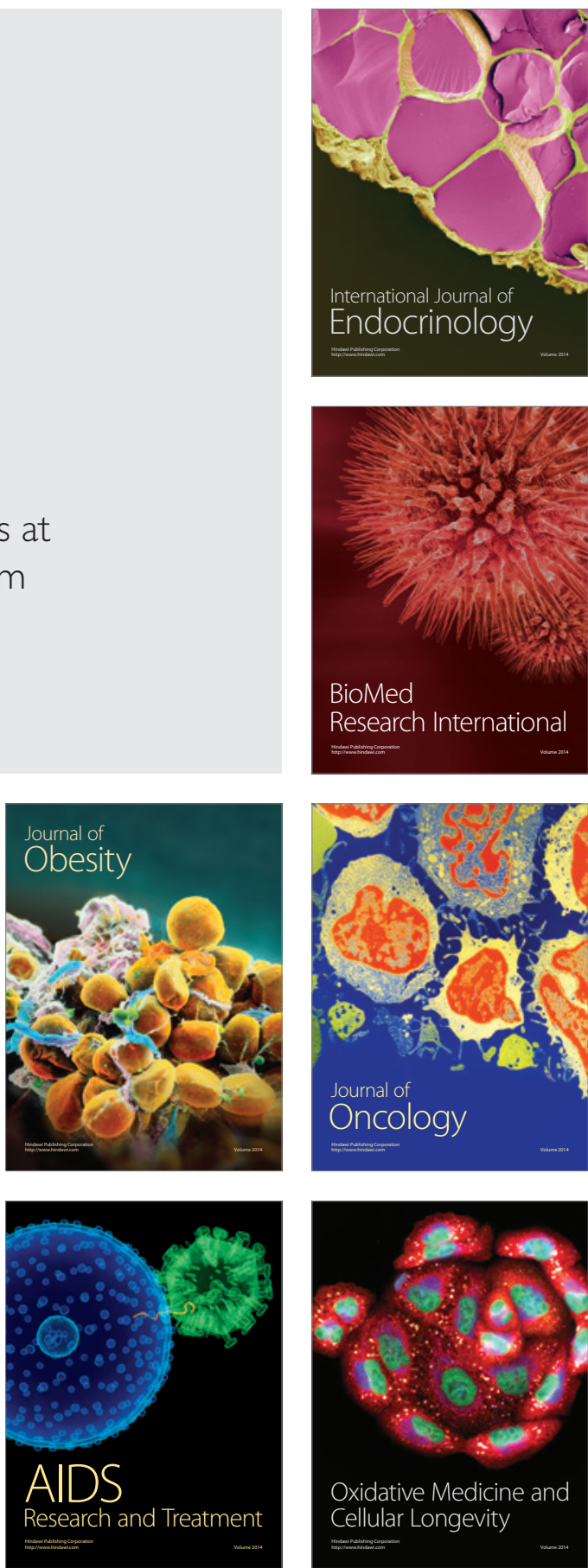